\title{
EXTRACTING AND ANALYSING DESIGN PROCESS DATA FROM LOG FILES OF ICT SUPPORTED CO-CREATIVE SESSIONS
}

\author{
Becattini, Niccolò (1); Cascini, Gaetano (1); O'Hare, Jamie Alexander (2); Morosi, Federico (1); \\ Boujut, Jean-Francois (3)
}

1: Politecnico di Milano; 2: University of Bath; 3: Grenoble Institute of Technology

\begin{abstract}
The observation of designers' behaviour in collaborative design activities and the analysis of protocols improved the understanding of how novel ideas emerge, what occurs among designers and, indirectly, what methods have a good impact on the outcomes. Yet, protocol analysis requires recording the design sessions, often in a simulated environment, thus introducing a bias in the observation. Moreover, the analysis takes up to 1000 times the duration of the observed design session. These limitations definitely hinder the scalability of this practice to large experiments in real operational environments.

This paper investigates the possibility to use the data collected in log files, automatically recorded during collaborative design sessions assisted by an ICT design support tool, as a means to extract relevant information about the design process and ultimately to infer insights about co-designers' cognition during the session. In this perspective, the paper proposes a set of metrics tailored to an Augmented Reality-based collaborative design tool. The study has been carried about by processing the data collected in 5 real case studies conducted in three different design companies.
\end{abstract}

Keywords: Human behaviour in design, Collaborative design, Big data, Design informatics, Design Protocol Analysis

Contact:

Becattini, Niccolò

Politecnico di Milano

Mechanical Engineering

Italy

niccolo.becattini@polimi.it

Cite this article: Becattini, N., Cascini, G., O'Hare, J.A., Morosi, F., Boujut, J.-F. (2019) 'Extracting and Analysing Design Process Data from Log Files of ICT Supported Co-Creative Sessions', in Proceedings of the 22nd International Conference on Engineering Design (ICED19), Delft, The Netherlands, 5-8 August 2019. DOI:10.1017/dsi.2019.16 


\section{INTRODUCTION}

The investigation of design processes in vivo, meant as the observation of designers' behaviour during their diverse activities, has become a widely adopted research method to obtain insights into how designers approach their tasks the thought processes that occur before a novel idea emerges. Unfortunately, there is plenty of evidence that this kind of investigation is also extremely resource demanding, both in terms of time and human resources (Blessing and Chakrabarti, 2009). In fact, for each hour spent designing, it takes 10 to 1000 hours to analyse one hour of recorded protocol (Jiang and Yen, 2009). Standard protocol analysis requires several activities to be done before delivering a reliable analysis of the observations. The data are typically qualitative, captured as videos and audio recordings of the design sessions, sometimes enriched with annotations by (external) observers. These qualitative data then have to go through initial processing, such as transcribing and segmenting into 'chunks', to generate a protocol that is ready for analysis. Typically, a coding scheme is then applied to the protocol, with validation of the coding being achieved through an inter-rater reliability test. Once complete, the coded data allows for qualitative and/or quantitative assessments (e.g. in terms of time spent for specific activities, in terms of recurring sequences of activities...) (Someren et al., 1994).

The clarity and robustness of the protocol is definitely the crucial factor that affects the efficiency of the whole process of analysis as this results in the mentioned very large time gap between the moment of the observation and the availability of the results. On top of this, the coding scheme requires, a sufficient amount of time for its development, its early testing and refinement so that it proves to be capable of capturing all the relevant factors to monitor and study during the observation (Becattini et al., 2018).

With a long term vision, one can expect that sooner or later it will be possible to run very fast, (semi-) automatic analysis of interactions, independently from the modality observed (gestural, spoken, mediated by design representations...), by making use of the rapidly improving capabilities of AI technologies leveraging motion capture and speech-to-text processing. From this perspective, we can envisage fitting participants with sensors, wearable technology or other sophisticated equipment so as to gather more objective and quantitative data ready to process. However, the intrusiveness of these devices might bias the recorded results as their presence might result in unnatural behaviour and make some (co-)designers feeling awkward (Peruzzini et al., 2018).

When studying design activities that involve participants interacting with an ICT system of some kind (e.g. CAD, sketching tools...), some features of the ICT system itself can be employed to assist with gathering of structured quantitative objective data about the behaviour of the user to make the gathering and analysis of data easier and faster.

Specifically, most of these systems create log files of all the operations carried out within the software application used for Human Computer Interaction (HCI). These log files are potentially a very valuable source of design behaviour data as they typically come in a semi-structured or easy to structure form (typically a text file with time stamps for every operation mentioned, organized in rows, one after the other). The increasing availability of computing power would suggest to use these data with a bottom up approach, so that the AI-based analysis of the data allows emergent behaviours to become evident. Despite this potential, log file data mining for design behaviour research has received little attention, with just few examples of log files used to infer information from the activities there noted and just peripheral contributions in the design domain.

According to the promising evidence mentioned in scientific literature, which are briefly reviewed in the next chapter, this paper aims at providing initial evidence of the kind of analysis supported log files analysis and how these data can provide significant evidence of what happened during the design process. The experimental setting used for this research is presented in Section 3, together with an initial set of indexes that aim at providing a first comprehensive representation of what happened during ICT-supported design sessions. The results from five real industrial case studies in the field of packaging and product interface design are presented in Section 4 and discussed with reference to the metrics. Section 5 discusses the result with reference to research objectives and highlights limitations and potential future developments for this kind of investigation. The last section concludes the paper with a summary of the contents and the main findings. 


\section{LOG FILE DATA: AN INFORMATION GOLDMINE IN MANY DOMAINS}

One of the first uses of log files to gather data about the user interaction with a hypertext dates back to 1992 (Carmel, 1992), but nowadays they are an extremely consolidated relevant data source in the ICT domain, as those data are typically considered vital in applications such as detection of intrusions within secured networks, attempts or some malicious behaviours (e.g. Patil et al., 2019). As log file data can support monitoring malicious behaviours, log files which map user interaction can, as a consequence also map and record user and machine behaviour in general. For instance, Landauer et al. (2018), made use of log file data about the activities users carried out to make it easier to identify repeated or sporadic actions. Within this trend of development, the increasing diffusion of apps boosted the importance of log files for troubleshooting the code as well as to monitor user behaviour (Kreiter, 2018), which is also an important factor to steer the development of their future features with smaller efforts by developers (Ferre et al., 2017). With a more abstract approach, Cattledge et al. (1995) focused on the access to the world wide web and used log files to infer browsing strategies during the interaction with a browser, which in turn results in the identification of typical behaviours emerging from log file data. Log files have also proved to contain relevant data about the usability of new technologies and their effects on users. For instance, Bouabid et al (2018) used log file analysis to grasp relevant insights about users' interaction with a platform for distant collaboration based on Tangible User Interfaces. These data have been proficiently combined with other data retrieved from interviews and direct observations in order to gain a comprehensive view.

Still with reference to the use of ICT system, but from a completely different perspective, literature is presenting a growing number of contributions where log files from e-learning platform are used in order to find correlations between the time spent in class, the time spent on the e-learning platform together with the activities there carried out and the final outcomes of the students attending Massive Open Online Courses (MOOCs), as well as different kinds of ICT supported distant learning (e.g. Theobald et al., 2018). From a similar angle, other scholars also addressed the analysis of log files in e-learning platforms in order to create typical user profiles and identify the most critical area where the HCI should be improved to facilitate users (Schultz et al., 2013). Within the analysis of the behaviour of MOOC users, Lee (2018) showed that, depending on the size and the contents of log files, these data can be used for sophisticated processing and analysis in order to identify emerging behaviours that are not evident otherwise (e.g. by means of clustering algorithms, machine learning etc.). Nevertheless, literature also witnesses that interesting insights do not necessarily emerge from purely quantitative analyses, as the qualitative analysis of log file data allows users' behaviour to be inferred so as to capture traits which are not easy to identify with a quantitative approach (Katai et al., 2012).

In the design context, there is no emerging contribution that deals with the analysis of log file data, despite some studies on log files from MOOCs and e-learning platforms investigated the learning outcomes in the field of Engineering Graphics (Wiebe et al., 2011), which has at least a weak link with design. This is also different from capturing data from email exchanges, e.g. as proposed in Snider et al. (2018), as the focus is just on communication in design. Some attempts to reduce the effort from protocol analysis by processing them almost automatically have been shown in the field of design both with traditional means (Becattini et al., 2014) and using data from ICT platforms (Becattini et al., 2012). Recent attempts tried to explore cognition with the support of data contained in log files, showing that this approach has a promising potential. For instance, such studies proved that log files data can provide an effective and efficient support for the assessment of spatial working memory capacity (Kornmann et al., 2016), of complex problem solving skills with very large scale studies (Greiff et al., 2015) as well as of the effects of stimuli when dealing with metacognitive learning (Bannert et al., 2015).

Consistently with the above literature-based evidences, the opportunities coming from the data contained in log files appear to be promising also to support the analysis of design protocols. The following sections aims at exploring and providing initial evidences to support the following research questions, so that further studies can be planned, if the early results are promising:

- Can log file data be used, as proficiently as in other domains, to gather relevant information about the design process? (i.e. could these data be correlated to typical behaviours in design cognition/design creativity?);

- What are the efforts required to run a log analysis in terms of time and human resources? 


\section{RESEARCH APPROACH}

\subsection{ICT tool to support designing}

According to the above premises, it appears quite clearly that there are just very few contributions which aim at using log files (or similar data sources) to infer what happened during design activities. In order to evaluate the viability of this approach and run a first estimation of the time and human resources involved, the authors opted to study collaborative design activities supported by an ICT tool that they are developing: the SPARK platform (SPARK project - http://spark-project.net - SPatial Augmented Reality as a Key for co-creativity). It exploits Spatial Augmented Reality (SAR, augmented reality displayed through projection) to support and foster collaborative creative thinking in the design process. The introduction of a mixed prototype - a tangible blank 3D shape and a virtual surface rendered on the shape by projection (Figure 1) - allows the participants to visualize a shared design representation of the solution and its evolution during the design process. This helps reduce language barriers, which can arise due to the diversity of backgrounds and level of sketching skills amongst the design team members.

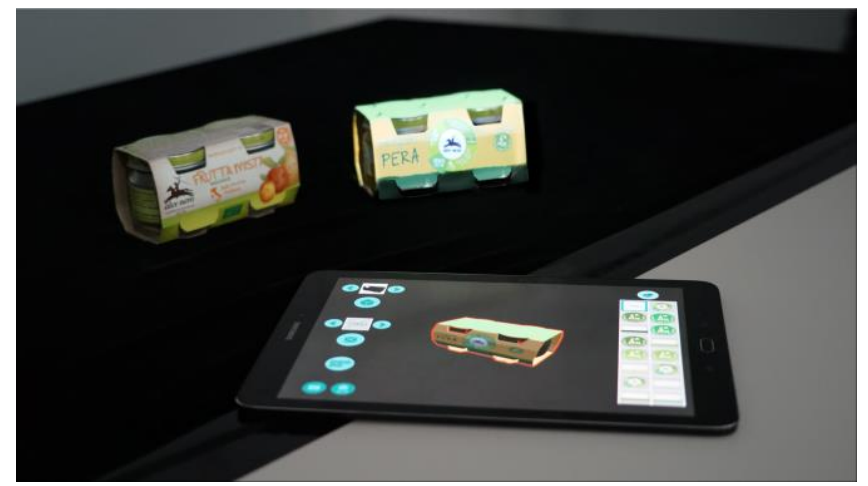

Figure 1: Some SPARK platform elements: a tablet with GUI to design onto the mixed prototype (above right), as rendered by projection. Real product on the left

The SPARK platform GUI enables co-designers to interact with digital content to project by introducing, removing, rearranging (in size, orientation, position and colour) items as images and texts, which renders surface finishes, materials, as well as buttons, handles/knobs, logos, product textual descriptions or evocative pictures.

An ICT tool to support design, as above described, allows the participants to avoid wearing invasive devices such as active goggles, whose prolonged presence over the users' head leads to discomfort and eyestrain. SAR technology is expected to be capable of significantly reducing the amount of iterations between two subsequent design meetings, as it enables to quickly switch and test a multitude of design alternatives during the co-creative design sessions. Through rapid iteration, review and filtering of design alternatives a significant reduction of time and human resources required per project can be achieved.

\subsection{Room setting and case studies in real operational environments}

The experimental sessions were conducted at the premises of Artefice and Stimulo (two design companies working in the field of packaging design and product design) and at Antwerp Management School, which has a unit to foster innovation by design; all the three entities are SPARK consortium partners. At these locations, rooms have been created so that the SPARK platform can work with complete functionalities, in a co-creation scenario that aims at facilitating the interaction, both with the mixed prototype and the media holding the SPARK platform GUI.

The design practitioners were able to facilitate the session as they wished, with no specific guidance from the authors of the research. Figure 2 shows the map of the SPARK rooms at Artefice, similar ones are installed at Stimulo and AMS. At Artefice, the SPARK GUI was run on a large (40" display) multi-touch screen, which allowed multiple designers to interact with the GUI. At the premises of Stimulo the SPARK GUI was controlled using a standard PC screen and mouse on screen which allowed designers and clients to visualize the GUI. At AMS, a standard (10" display) touchscreen tablet-PC was used. Five case studies have been tested in these settings: 2 at Artefice, 2 at Stimulo, 1 at AMS, as detailed in Table 1. 
Food Inc. (name anonymised for commercial confidentiality reasons) is a food manufacturer that specialises in high quality, food products and condiments. Food Inc. have an on-going project with Artefice to design packaging for a new product. The main objective of the session was to present the rework done on the packaging following an earlier session (completed without the use of the SPARK Platform). This case study comprises two subsequent co-creative design sessions before the final confirmation of the packaging proposal.

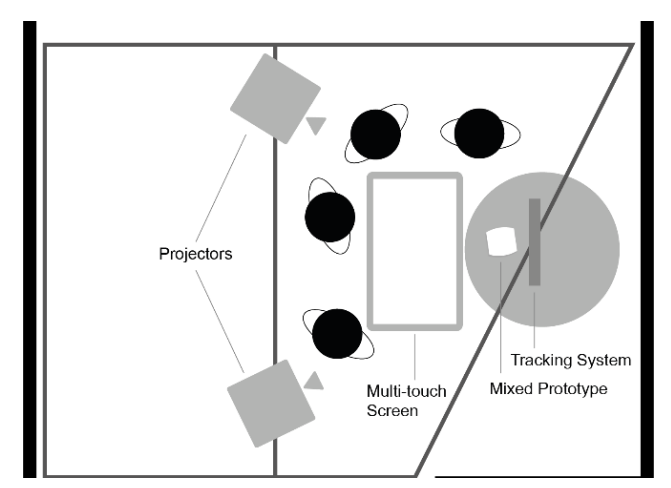

Figure 2: Top view of one of the SPARK room (Artefice)

Zobele Group is a world leader in the business segments of 'Air and 'Pest Control' and is also a global player in the 'Health \& Personal Care' and 'Fabric \& Laundry Care' markets. The session explored concepts for Zobele's products packaging so as to also appreciate the SPARK platform functionalities. Wavecontrol is an engineering company, founded in 1997 and specialising in the industrial products for the measurement of electromagnetic fields. The purpose of the session was to create new ideas for a new range of products which requires designing the product user interface.

Samsonite is an American luggage manufacturer and retailer, with products ranging from large suitcases to small toiletries bags and briefcases. During the session multiple design concepts for each Samsonite suitcase are presented on a 1:1 scale foam model and final design decisions on the product aspect are made.

Food Inc. and Zobele are, therefore, considered as case studies about packaging design, while Wavecontrol and Samsonite as product (interface) design.

Table 1: Summary of the co-creative sessions considered for log file analysis

\begin{tabular}{|c|l|c|c|}
\hline Company and topic & Host & $\begin{array}{c}\text { Objectives of the co-creative } \\
\text { sessions }\end{array}$ & Participants \\
\hline $\begin{array}{c}\text { New product packaging } \\
\text { (2 sessions) }\end{array}$ & Artefice & $\begin{array}{c}\text { Explore attractive and effective } \\
\text { variants from previous concepts and } \\
\text { converge to a final version }\end{array}$ & $\begin{array}{c}\text { Artefice's designers and } \\
\text { Food Inc. top } \\
\text { management }\end{array}$ \\
\hline $\begin{array}{c}\text { Zobele } \\
\text { Fragrance packaging }\end{array}$ & Stimulo & $\begin{array}{c}\text { Check feasibility of using } \\
\text { miniSPARK for showcasing their } \\
\text { products and variants at trade shows }\end{array}$ & $\begin{array}{c}\text { Stimulo's designers + } \\
\text { Zobele's design } \\
\text { managers }\end{array}$ \\
\hline $\begin{array}{c}\text { Wavecontrol } \\
\text { Real-Time Handheld } \\
\text { Analyzer device }\end{array}$ & Stimulo & $\begin{array}{c}\text { Test user interaction aspects of the } \\
\text { large touch screen that appears on } \\
\text { the product }\end{array}$ & $\begin{array}{c}\text { Stimulo's designers + } \\
\text { Wavecontrol's directors } \\
\text { and technical managers }\end{array}$ \\
\hline $\begin{array}{c}\text { Samsonite NV } \\
\text { models of luggage } \\
\text { suitcases }\end{array}$ & AMS & $\begin{array}{c}\text { Check the performance of the } \\
\text { SPARK platform for use in design } \\
\text { review situations }\end{array}$ & $\begin{array}{c}\text { AMS staff + top } \\
\text { management of } \\
\text { Samsonite }\end{array}$ \\
\hline
\end{tabular}

\subsection{Metrics for the analysis of log file data}

The log file records every function the users carry out with the platform. This includes the changes made to the canvas that contains the textual/graphical contents to project (position, size, orientation, layer level for asset or group of assets), the background colour of the mixed prototype, as well as its spatial characteristics within the user interface. These data provide quantitative information about the outcomes of the session with an objective measurement of the whole process followed by the co-designers. Data 
are collected with time stamps, so that it is also possible to measure durations and get insights about the process and its progress.

The data collected in the log files are to be processed to extract the indexes proposed in this paper and presented in Table 2. These data contribute to answer the first research question stated in section 2: "Can $\log$ file data be used, as proficiently as in other domains, to gather relevant information about the design process? Could these data be correlated to typical behaviours in design cognition/design creativity?"

Therefore, the following indexes relates to typical design creativity and cognition constructs, such as: quantity of design moves (e.g. Lee et al., 2003) here referable to \#Funct and \#Funct_eff; quantity of ideas and exploration of the design space/variety of solutions (e.g. Shah et al., 2003) here referable to \#Asset and \#Variant; duration of sessions (e.g. Saliminamin et al., 2018) and frequency of the design moves (Kan and Gero, 2009) which here respectively point to \#Time and \#Effectiveness. Moreover, the data collected allow for a preliminary estimation of the effectiveness of the interaction with the GUI by co-designers, which is an (in)direct evidence of the impact of the technology on the design process (\#HCI_GUI), which looks into a similar direction of what Rahimian and Ibrahim (2011) observed.

Table 2: Indexes to measure design process data contained in the log files analysed

\begin{tabular}{|l|l|l|l|}
\hline \multicolumn{1}{|c|}{ Index } & \multicolumn{1}{|c|}{ Label } & \multicolumn{1}{c|}{ Processed data } & \multicolumn{1}{c|}{ Quantitative evidence of... } \\
\hline $\begin{array}{l}\text { Number of functions } \\
\text { initiated or continued } \\
\text { in the log file }\end{array}$ & \#Funct & $\begin{array}{l}\text { Number of rows in the log } \\
\text { file }\end{array}$ & $\begin{array}{l}\text { Number of activities/design } \\
\text { moves within the session }\end{array}$ \\
\hline $\begin{array}{l}\text { Number of effective } \\
\text { functions }\end{array}$ & \#Funct_eff & $\begin{array}{l}\text { Switches between different } \\
\text { functions and functions } \\
\text { repeated after 3 seconds }\end{array}$ & $\begin{array}{l}\text { Amount of changes made to the } \\
\text { design - mixed prototypes used } \\
\text { for evaluation }\end{array}$ \\
\hline $\begin{array}{l}\text { Number of assets } \\
\text { used within the } \\
\text { sessions }\end{array}$ & \#Asset & $\begin{array}{l}\text { Number of previously } \\
\text { prepared digital contents } \\
\text { used within the sessions }\end{array}$ & $\begin{array}{l}\text { Number of items (images, text) } \\
\text { used for the interaction with the } \\
\text { mixed prototype }\end{array}$ \\
\hline $\begin{array}{l}\text { Number of solution } \\
\text { variants potentially } \\
\text { explored }\end{array}$ & \#Variant & $\begin{array}{l}\text { Number of functions } \\
\text { involving \#asset and changes } \\
\text { to background colour of the } \\
\text { prototype }\end{array}$ & $\begin{array}{l}\text { Number of solution variants } \\
\text { tested/checked during the } \\
\text { session }\end{array}$ \\
\hline Duration & $\begin{array}{l}\text { Difference between start and } \\
\text { end time (seconds) }\end{array}$ & $\begin{array}{l}\text { The time required to run co- } \\
\text { creative sessions (to be referred } \\
\text { to \#variants to check the } \\
\text { efficiency of the session) }\end{array}$ \\
\hline Session Effectiveness & \#Effectivene \\
ss & = \#Time / \#Variant & $\begin{array}{l}\text { The time required to switch } \\
\text { from a variant to a next one }\end{array}$ \\
\hline HCI-GUI efficiency & \#HCI_GUI & = \#Funct_eff / \#Funct & $\begin{array}{l}\text { The efficiency of the SPARK } \\
\text { UI in terms of No of changes } \\
\text { made to the design with respect } \\
\text { to the No of activities requested } \\
\text { to the interacting user(s) }\end{array}$ \\
\hline
\end{tabular}

\section{RESULTS OF THE LOG FILE ANALYSIS}

The log files of the sessions listed in Table 1 have been considered for the analysis, according to the metrics presented in Section 3.3. The resulting values are shown in Table 3.

As a starting point, it is worth noticing that the evidences gathered regards a sufficiently representative set of case studies in terms of duration: from approximately 30 minutes to more than 2 hours. This is also true in terms of the size of the mixed prototype. While the two packaging design sessions focused on solutions whose size is enclosed into a cube of maximum $10 \mathrm{~cm}$, the product design sessions shifted to devices which were larger in size (to the extent of a full size suitcase).

This marked difference is also shown by the duration of the sessions (\#Time), which has higher consistency within the design domain (longer durations for product interface design, generically shorter for packaging design). \#Time is also not correlated with the amount of functions used during the sessions, both considering \#Funct and \#Funct_eff. This suggest that also the amount of options selected during the sessions are strongly case study dependent. 
Table 3. Results of the log file analysis for the product and packaging design sessions

\begin{tabular}{|l|l|l|l|l|l|l|l|}
\hline PROD. & \#Funct & \#Funct_eff & \#Asset & \#Variant & \#Time & \#Effectiveness & \#HCI_GUI \\
\hline Samsonite & 1777 & 346 & 13 & 186 & $6074 \mathrm{~s}$ & $32.7 \mathrm{~s}$ & $19,5 \%$ \\
\hline Wavecontrol & 3634 & 602 & 17 & 412 & $8212 \mathrm{~s}$ & $19,9 \mathrm{~s}$ & $16,6 \%$ \\
\hline PACK. & \#Funct & \#Funct_eff & \#Asset & \#Variant & \#Time & \#Effectiveness & \#HCI_GUI \\
\hline Food Inc. 1 & 1101 & 472 & 37 & 377 & $3839 \mathrm{~s}$ & $10,2 \mathrm{~s}$ & $42,9 \%$ \\
\hline Food Inc. 2 & 741 & 229 & 17 & 140 & $1828 \mathrm{~s}$ & $13,1 \mathrm{~s}$ & $30,9 \%$ \\
\hline Zobele & 2378 & 482 & 18 & 354 & $2477 \mathrm{~s}$ & $7 \mathrm{~s}$ & $20,3 \%$ \\
\hline
\end{tabular}

As previously said, the two sessions of the Food Inc. case study should be considered as sessions of the same project. \#Time of session 2, in fact, is approximately one half of session 1, as most of the design was decided after the first session and required just final confirmation. The same ratio is confirmed by the \#Assets used in the two sessions and the \#Variants generated, evaluated and refined. This shows that the SPARK platform allows co-designer to converge quickly on design concepts and ideas that have been previously developed within the same project, substantially confirming its expected potential to slash down the amount of time and human resources per project in design companies (see Section 3.1).

Differences among these case studies are also evident when one compares the \#Assets and the \#Variants. This said, it is generally true that all the sessions show significantly high values of \#Variants checked during the sessions. This means that both the product design and packaging design sessions display that the SPARK platform actually supports the evaluation of hundreds of different alternatives in a very short time: the Wavecontrol session has the higher \#Variant score among the sessions (412), while the Food Inc 2 has the lowest (140), which remains a significantly high value with reference to the nature of that session, as mentioned above. This is also an indirect evidence of the extent of creative exploration of design alternatives in co-creative sessions.

These values are also confirmed by the ease of application of changes, as witnessed by \#Effectiveness: to switch from a prototype variant to another one, the co-designers had to "wait" from 7 to approximately 30 seconds. These values are also significant with reference to the time required to prepare real-like physical prototypes as the SPARK mixed ones aim at substituting. \#Effectiveness, as said in section 3.3, represent the average amount of seconds in between the appearance of design modifications to the prototype (\#Time/\#Variant). In these terms, it appears evident that some of the considered changes should occur very quickly (in any case more than 3 seconds to be accounted by the metrics), as sometimes \#Efficiency scores below 10 seconds. This means that just part of the \#Variants have been considered for evaluation and that some intermediate steps just aimed at building a comprehensive design proposal, sufficiently detailed to run a meaningful assessment of its suitability. Nevertheless, this is a strong evidence that the amount of time required to modify the mixed prototype does not represent a bottleneck for the usage of the technology, suggesting that it reaches the expected effectiveness in terms of potentiality. The results concerning the efficiency of use of the GUI/HCI are also extremely interesting, as they account for the amount of functions carried out with a satisfactory result (\#Funct_eff considers, functions ended with, e.g., the proper placements, orientation and size of assets) with reference to the complete set of functions used along the session (\#Funct, thus including those for which the co-designers had to refine what done in a very short time, namely below 3 seconds). The resulting percentages provide meaningful results to highlight how much the differences in setting and technical equipment for the companies involved affect the results.

Food Inc. sessions were carried out at Artefice, whose SPARK room is equipped with a large touch screen for HCI (\#HCI_GUI values: 30-40\%), while co-designers in the Zobele, Wavecontrol and Samsonite sessions used tablets or mouse and keyboard (\#HCI_GUI values around 15-20\%) as for the SPARK rooms equipped at Stimulo and AMS. These figures suggest that a large interaction surface supports a more efficient selection of functions, probably due to an increased precision of placement and command execution. A detailed analysis of \#HCI_GUI per function (number of satisfactory use of the function/overall amount of use of the function) goes beyond the purpose of this study.

Compared to the values of Table 3, which just provide a comprehensive description of the design session, the curves in Figure 3 show how the co-designers spent their time during the 5 co-creative design sessions. These graphs, show how much time the participants spent on each design move, from the beginning to the end. Steeper curve sections correspond to more rapid changes to the design proposal/mixed prototype, while flat portions of the curve correspond to pauses with the SPARK GUI. 
These pauses could be due to different reasons, which are not directly inferable from the log file data (from breaks to discuss the proposed solutions before applying new changes to the prototype, to simply coffee breaks). These curve also show that, despite the large variability of case studies, there are some evident regularities. Beyond minor fluctuations, most of the considered curves show a very similar angular coefficient in the timeframes where the curve is not flat. Samsonite could be an exception, but beyond more frequent breaks (higher presence of horizontal curve portions), the inclination of steep traits is very close to the other ones. These regularities are likely to be dependent on the technology, as it was the only factor the 5 sessions shared.

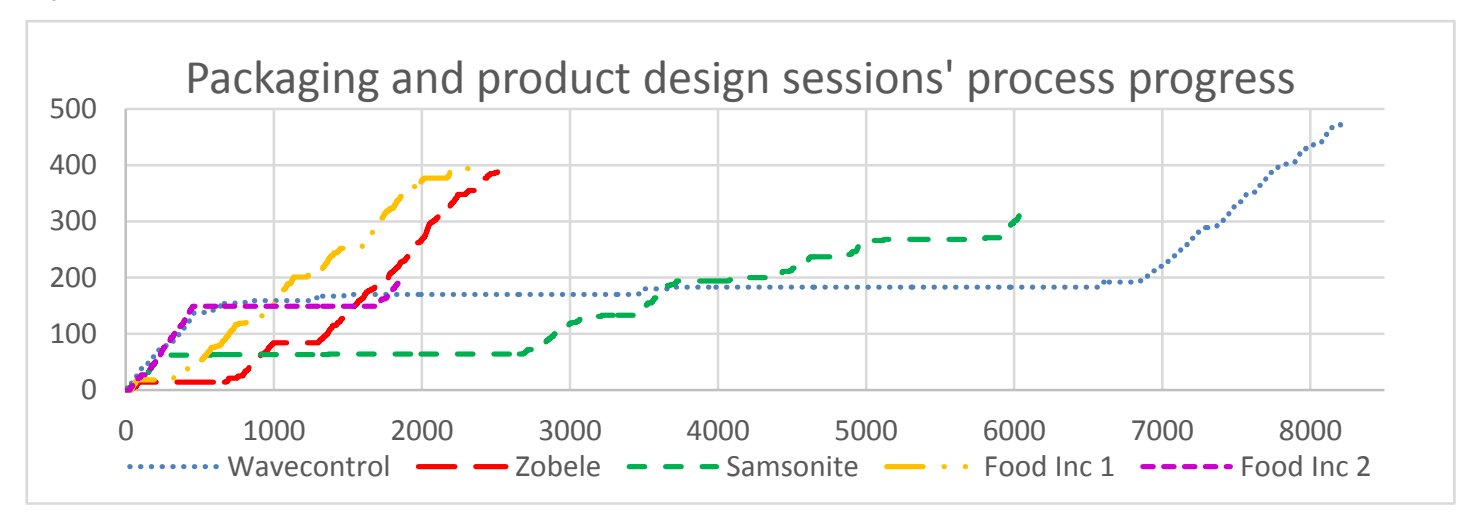

Figure 3: curves of cumulative time spent designing (x-axis) with regards to the number of design moves ( $y$-axis)

\section{DIscussion}

The results presented and commented in Section 4 provide a preliminary evidence that log files data have a potential to gather relevant information also in the design domain. In fact, the whole set of 5 case studies allowed log file data to be processed according to an originally developed metrics focusing on design cognition and creativity concepts, such as fluency of idea generation and exploration of the design space. The data contained in the log files allowed to easily quantify representative aspects of design sessions. The indexes, as well, allow to run comparisons and appreciate differences/similarities among sessions. In detail, the data contained in log files showed how many design moves co-designers made during a design session, how many solution alternatives (partial or complete) they have generated and viewed for a possible evaluation, how much time they spent for the whole design process, how much time they spent interacting with the ICT tool to support design activities.

Beyond what the metrics is explicitly capable of identifying, the comparisons between/among sessions that belong to the same design project, as for instance the Food Inc case study, makes it possible to appreciate evidence of convergence of the whole design process. In fact, indexes that show reduced durations and smaller numbers of generated/evaluated alternatives, along subsequent sessions of the same project, highlight the convergence towards a unique solution. This is consistent with most of the literature describing the design process. In addition, the fact that this finding is not directly emerging because of the metrics, but through the metrics (with further processing), provides a further strong evidence towards the suitability of the approach to get meaningful insights about various facets of the design process. Beyond the research questions addressed in this paper, the analysis of log file data also allows us to gather interesting and useful information about the ICT tool used to support the design activity. The 5 SPARK Project case studies used for this investigation were carried out under very similar conditions, except for the devices they used to communicate with the SPARK platform server and to interact with the other co-designers (a PC with mouse and keyboard, a tablet, a very large touchscreen). The processed data show that larger surfaces made the participants more capable of applying modification efficiently, with a smaller number of repeated moves to adjust what was not satisfactorily placed on the design canvas. In itself, despite the specificity of the technology used across the 5 case studies, this could be considered a conjecture this study allowed to formulate and that becomes interesting to verify with other ICT tools (e.g. sketching tools, CAD/CAE systems...), which require interacting with a screen of various sizes.

The analysis of the design process progress also showed a very interesting and unexpected result: independently from the design domain and from the duration of breaks, all the periods of activities where people interacted with the SPARK platform had approximately the same steepness. 
For what concerns the amount of human resources and time required to run this study on log files, it is worth to say that the direct comparison with design protocol analysis should consider that the starting point is extremely different in these two scenarios. Design protocol analysis requires multiple coders to go through the qualitative data contained in recordings, transcribe them into a protocol and label its segments. This is to achieve results that are significantly homogeneous among the individually coded protocols, to avoid an excess of subjectivity. The quantitative and objective nature of the data contained in the $\log$ files substantially reduces the need to turn qualitative into quantitative data or to make them as objective as possible. The whole process of analysis for the $5 \log$ files data (provided in rows as comma separated values) required approximately 5 hours for the definition of spreadsheet rules to process data and extract the required indexes. Then, every log file required approximately 1 hour to apply the rules to calculate indexes and to extract graphs and other relevant information. The overall duration of the 5 case studies is 6 hours and 15 minutes and their analysis required one investigator to work for about 10 hours in total. Standard design protocol analysis requires, according to the experience of the authors in the same project, approximately 60 hours per hour of recording, which is consistent with what stated in the introduction (1:10-1000). The ratio "time for the analysis: time of the observation" with log file analysis, on the contrary drops to a much more convenient value: 1,6:1. This difference can be meaningful just in case further studies demonstrate that the analysis of $\log$ files can actually substitute more traditional design protocol analysis. The self-sufficiency of the $\log$ files for these studies, however, is a visionary perspective, so far. In fact, the curves shown in Figure 3 highlight that log files are capable of describing process data, but it is still impossible to distinguish what is happening during the design session when people do not interact with the GUI (flat segments). Nevertheless, this does not exclude that log files could become useful to drive the identification of more relevant parts of the protocol to analyse with standard approaches as, on the one hand, pauses or, on the other hand, period of frequent changes to the design solution. Further investigations will be required to clarify what role log files can play for the analysis of cognition and creativity, but these results suggest this is a promising direction.

In general, this approach has a much stronger potential to run studies involving subjects on a large scale, which is unconceivable for design protocol analysis because of the time and human resources it requires. As mentioned above, an approach based on log file data allows to generate results more easily and rapidly, but just with partial details, as the log file only captures details of user interaction the ICT tool.

\section{CONCLUSION}

This paper investigates if data contained in log files, recorded during collaborative design sessions assisted by an ICT design support tool, are suitable to provide relevant information about the design process, with the purpose of inferring relevant insights about co-designers' cognition and creativity expressed during the session. The data from 5 different real industrial case studies show that the approach is suitable to extract design-relevant information from data just with the application of a coarse grained metrics. This kind of analysis has also proved to be extremely effective to provide results in a short time, as log file contains objective data and do not require multi-rater coding to determine their reliability. With respect to standard protocol analysis, it is still necessary to run tailored investigation in order to compare the outcomes these two approaches produce, especially in terms of contents. This comparison should provide new evidences to determine if log file data can partially substitute design protocol analysis, complement its results or simply helps researchers in spotting the most relevant set of segments to analyse, in order to improve the efficiency of what is otherwise a very time intensive task.

\section{ACKNOWLEDGEMENT}

This paper exploits data collected within the SPARK project, which has received funding from the European Union's Horizon 2020 research and innovation programme under GA No.688417. This paper reflects only the authors' views and the European Commission is not responsible for any use that may be made of the information it contains.

\section{REFERENCES}

Bannert, M., Sonnenberg, C., Mengelkamp, C. and Pieger, E. (2015), "Short-and long-term effects of students' self-directed metacognitive prompts on navigation behavior and learning performance." Computers in Human Behavior, Vol. 52, pp. 293-306. 
Becattini, N., Cascini, G., O’Hare, J.A. and Masclet, C. (2018), “Coding schemes for the analysis of ICT supported co-creative design sessions." In DS92: Proceedings of the DESIGN 2018 15th International Design Conference (pp. 533-544).

Becattini, N., Cascini, G. and Rotini, F. (2015), An OTSM-TRIZ based framework towards the computer-aided identification of cognitive processes in design protocols. In Design Computing and Cognition'14 (pp. 99-117). Springer, Cham.

Becattini, N., Borgianni, Y., Cascini, G. and Rotini, F. (2012), Experiencing protocol analysis of computerguided design tasks. In DS 70: Proceedings of DESIGN 2012, the 12th International Design Conference, Dubrovnik, Croatia (pp. 1821-1830).

Bouabid, A., Lepreux, S. and Kolski, C. (2018), "Study on generic tangible objects used to collaborate remotely on RFID tabletops." Journal on Multimodal User Interfaces, pp. 1-20.

Blessing, L. T., and Chakrabarti, A. (2009). DRM, a design research methodology. Springer Science \& Business Media.

Carmel, E., Crawford, S. and Chen, H. (1992), "Browsing in hypertext: A cognitive study." IEEE transactions on Systems, Man, and Cybernetics, Vol. 22, No. 5, pp. 865-884.

Catledge, L.D. and Pitkow, J.E., 1995. Characterizing browsing behaviors on the world-wide web. Georgia Institute of Technology.

Ferre, X., Villalba, E., Julio, H. and Zhu, H. (2017), September. "Extending Mobile App Analytics for Usability Test Logging." In IFIP Conference on Human-Computer Interaction (pp. 114-131). Springer, Cham.

Greiff, S., Wüstenberg, S. and Avvisati, F. (2015), "Computer-generated log-file analyses as a window into students' minds? A showcase study based on the PISA 2012 assessment of problem solving." Computers \& Education, Vol. 91, pp. 92-105.

Jiang, H. and Yen, C.-C. (2009), rotocol analysis in design research: a review. In Rigor and Relevance in Design: IASDR 2009, Seoul, Korea October 18-22, Seoul, pp. 147-156. International Association of Societies of Design Research.

Kan, J.W. and Gero, J.S. (2009), Using the FBS ontology to capture semantic design information in design protocol studies. In About: Designing. Analysing Design Meetings (pp. 213-229). CRC Press.

Kafai, Y. and Fields, D. (2012), Connecting play: Understanding multimodal participation in virtual worlds. In Proceedings of the 14th ACM international conference on Multimodal interaction (pp. 265-272). ACM.

Kornmann, J., Kammerer, Y., Anjewierden, A., Zettler, I., Trautwein, U. and Gerjets, P. (2016), "How children navigate a multiperspective hypermedia environment: The role of spatial working memory capacity." Computers in human behavior, Vol. 55, pp. 145-158.

Krieter, P. and Breiter, A. (2018), Analyzing mobile application usage: generating log files from mobile screen recordings. In Proceedings of the 20th International Conference on Human-Computer Interaction with Mobile Devices and Services (p. 9). ACM.

Landauer, M., Wurzenberger, M., Skopik, F., Settanni, G. and Filzmoser, P. (2018), Dynamic log file analysis: An unsupervised cluster evolution approach for anomaly detection. computers \& security, 79, pp.94-116.

Lee, G., Eastman, C.M. and Zimring, C. (2003), "Avoiding design errors: a case study of redesigning an architectural studio.” Design Studies, 24(5), pp.411-435.

Lee, Y., 2018. "Using Self-Organizing Map and Clustering to Investigate Problem-Solving Patterns in the Massive Open Online Course: An Exploratory Study.” Journal of Educational Computing Research, p. 0735633117753364.

Patil, D.N. and Meshram, B.B. (2019), Web Browser Analysis for Detecting User Activities. In Recent Findings in Intelligent Computing Techniques (pp. 279-291). Springer, Singapore.

Peruzzini, M., Grandi, F. and Pellicciari, M. (2018), "How to analyse the workers' experience in integrated product-process design.” Journal of Industrial Information Integration, Vol. 12, pp. 31-46.

Rahimian, F.P. and Ibrahim, R. (2011), "Impacts of VR 3D sketching on novice designers' spatial cognition in collaborative conceptual architectural design.” Design Studies, Vol. 32, No. 3, pp. 255-291.

Saliminamin, S., Becattini, N. and Cascini, G. (2018), "Sources of creativity stimulation for designing the next generation of technical systems: correlations with R\&D designers' performance." Research in Engineering Design, pp. 1-21.

Someren, M. V., Barnard, Y. F., and Sandberg, J. A. ((1994), The think aloud method: a practical approach to modelling cognitive processes. Academic Press.

Schulz, A.H. and Breiter, A., 2012, August. Monitoring user patterns in school information systems using logfile analysis. In IFIP Conference on Information Technology in Educational Management (pp. 94-103). Springer, Berlin, Heidelberg.

Snider, C., Škec, S., Gopsill, J.A. and Hicks, B.J. (2017), The characterisation of engineering activity through email communication and content dynamics, for support of engineering project management. Design Science, 3.

Theobald, M., Bellhäuser, H. and Imhof, M. (2018), "Identifying individual differences using log-file analysis: Distributed learning as mediator between conscientiousness and exam grades." Learning and Individual Differences, Vol. 65, pp.112-122. 